\title{
An Analytical Solution for Transient Heat Conduction in a Composite Slab with Time-Dependent Heat Transfer Coefficient
}

\author{
Ryoichi Chiba $(\mathbb{D}$ \\ Department of Mechanical Systems Engineering, National Institute of Technology, Asahikawa College, 2-2-1-6 Shunkodai, \\ Asahikawa 071-8142, Japan \\ Correspondence should be addressed to Ryoichi Chiba; chiba@asahikawa-nct.ac.jp
}

Received 17 December 2017; Accepted 12 March 2018; Published 19 April 2018

Academic Editor: Filippo de Monte

Copyright (C) 2018 Ryoichi Chiba. This is an open access article distributed under the Creative Commons Attribution License, which permits unrestricted use, distribution, and reproduction in any medium, provided the original work is properly cited.

\begin{abstract}
An analytical solution is derived for one-dimensional transient heat conduction in a composite slab consisting of $n$ layers, whose heat transfer coefficient on an external boundary is an arbitrary function of time. The composite slab, which has thermal contact resistance at $n-1$ interfaces, as well as an arbitrary initial temperature distribution and internal heat generation, convectively exchanges heat at the external boundaries with two different time-varying surroundings. To obtain the analytical solution, the shifting function method is first used, which yields new partial differential equations under conventional types of external boundary conditions. The solution for the derived differential equations is then obtained by means of an orthogonal expansion technique. Numerical calculations are performed for two composite slabs, whose heat transfer coefficient on the heated surface is either an exponential or a trigonometric function of time. The numerical results demonstrate the effects of temporal variations in the heat transfer coefficient on the transient temperature field of composite slabs.
\end{abstract}

\section{Introduction}

Generally, heat transfer coefficients (HTCs) vary with time, and their variations can be random or, in certain cases, periodic. For example, because of irregular fluid motion around turbine blades, the HTC of the blade surfaces fluctuates [1]. The surface HTCs of the fuel elements in a boiling water reactor and a pebble bed reactor also vary over time [2]. The same holds for the HTCs between a casting and its metal moulds [3], on diesel fuel droplets subjected to transient heating [4], and on solids enveloped by pulsating flows of liquid or gas in internal combustion engines [5]. In addition, during the thermal processing of foods, the pattern of air circulation may be altered around them, resulting in changes of the surface HTC [6]. The time variation in the surface aspects of objects is also one of the possible reasons (e.g., oxidation, dust contamination, and fissuring) [7]. Such a temporal change in the HTC leads to alterations in the object's temperature.

Transient heat conduction with boundary conditions including a time-dependent HTC has been investigated since the late 1960s. Studies using (semi)numerical methods include the combined use of the finite difference and Laplace transform methods [8] and the parameter-group transformation followed by the Runge-Kutta shooting method [9]. Early studies using approximate methods include those of Ivanov et al. [10-12], which transformed the governing equation into a nonlinear equation using the change of variables and omitted the nonlinear term from the derived equation by restricting the research object to thin bodies. Kozlov [13] reduced the original problem to finding a solution to an infinite number of simultaneous ordinary differential equations, approximating it by a finite number. In addition, the application of the Laplace-Carson integral transform [7], finite integral transform methods with time-dependent eigenvalues [14, 15], and Lie point symmetry analysis [16] were reported. Further, an analytical method based on the Laplace transform and bifrequency transfer function was presented, but the inverse transform is extremely difficult [17]. An analytical solution to the heat conduction problem with arbitrary time-dependent HTC at the boundary had not been obtained for a long period because of the difficulty 
posed by eigenfunctions and eigenvalues that depend on time. However, Lee and colleagues [18] resolved this situation in 2010; they successfully derived explicit-form solutions by means of the shifting function method $[19,20]$.

On the other hand, there have been few studies with composite media. Il'chenko [21] obtained an approximate solution to the heat conduction problem of a two-layered plate with time-dependent HTC by replacing the continuous time function of the HTC with a piecewise function. Prikhod'ko [22] derived a transient temperature solution of a two-layer plate using a special series expansion of Green's function, while assuming that the temperature is uniform across the thickness in one layer. Yener and Ozisik [23] extended an analytical method based on a finite integral transform [14] to solve the problem for finite composite media with variable boundary condition parameters. All of them are, however, approximate methods, leading to less rigorous analysis.

Composite (or multiregion) media have a wide application in many industrial, environmental, and biological fields (e.g., in turbines, heat exchangers, fuel cells, and brake systems). Thus, it is industrially important to study this kind of heat conduction problems for composite media. To be specific, the need to analyse multiregion problems including contact resistances has been underlined owing to the canning of fuel elements in a reactor [24] and design of thermal insulation systems $[21,22]$. In those systems, ambient temperature may vary with time as well as HTC. In studying such engineering problems, analytical solutions are highly valuable as they provide greater insight into the solution behaviour, which is typically missing in any numerical solution. They can be used to benchmark numerical solutions as well. The foregoing facts motivate the present study.

In this paper, we address the one-dimensional transient heat conduction problem of an infinite composite slab with general time-dependent HTC at one external boundary. The shifting function method developed by Chen et al. [18] and an orthogonal expansion method $[25,26]$ are used to obtain an analytical solution for the multiregion heat conduction caused by time-varying ambient temperatures. Main differences from the approach for single layered slab [20] are the initial shifting of solution in each layer, the addition of interface conditions, and the eigenfunction expansion of functions based on the Vodicka type of orthogonality relationship, which would be the novelty of the present analytical method. Numerical calculations are performed to verify the solution for several test cases. Subsequently, two numerical examples are given to quantify the effects of the temporal variations in the HTC on the temperature profiles in the slabs.

\section{Problem Formulation}

Let us consider a composite slab consisting of $n$ layers, as shown in Figure 1, which has finite thickness $H$ but extends to infinity in the other two dimensions. The composite slab has an arbitrary initial temperature distribution $T_{\text {in }}(z)$ and is subjected to two different, arbitrary time-varying ambient temperatures, $y_{1}(t)$ and $y_{2}(t)$, at its external boundaries.

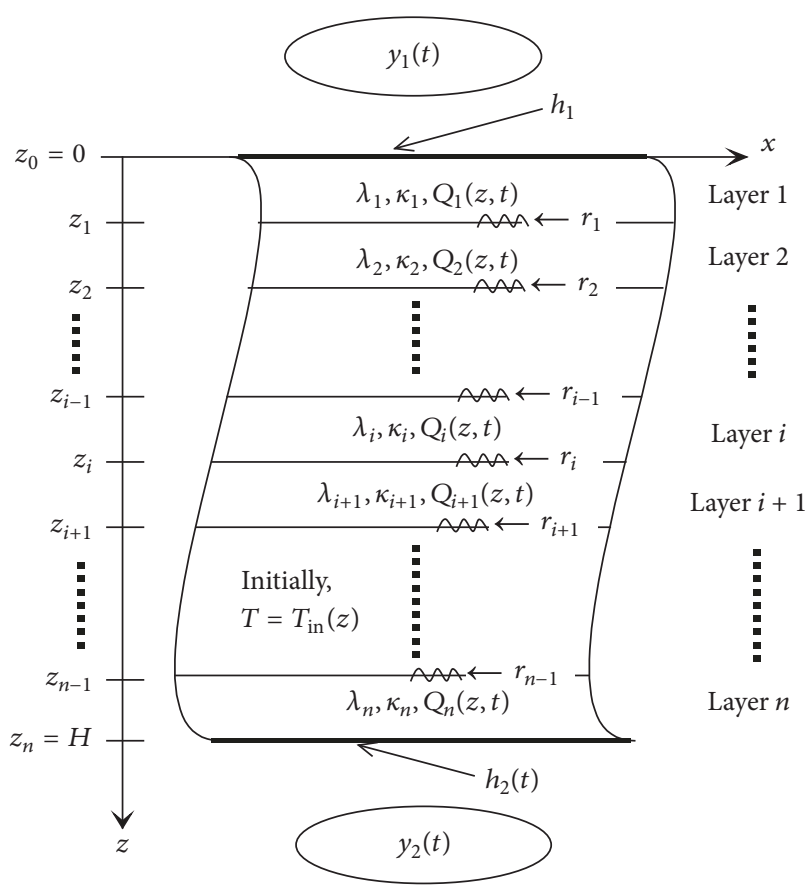

Figure 1: Schematic of an infinite composite slab with a timedependent heat transfer coefficient on one external boundary.

The boundaries have different HTCs, $h_{1}$ and $h_{2}(t)$, one of which depends on time. At the $n-1$ interfacial boundaries, heat is transferred through different constant conductance coefficients $r_{i}$, under the condition of heat flux continuity. A time- and space-dependent internal heat generation $Q_{i}(z, t)$ is also considered in each layer. The material of each layer is isotropic and homogeneous with constant thermophysical properties.

The temperature distribution in the $i$ th layer of $n$ solidly joined layers is given by the diffusion equation:

$$
\frac{1}{\kappa_{i}} \frac{\partial T_{i}}{\partial t}=\frac{\partial^{2} T_{i}}{\partial z^{2}}+\frac{Q_{i}(z, t)}{\lambda_{i}} \text { for } i=1,2, \ldots, n .
$$

This composite slab is subject to the following initial and external boundary conditions:

$$
\begin{gathered}
T_{i}=T_{\text {in }}(z) \\
\text { at } t=0 \text { for } i=1,2, \ldots, n, \\
\lambda_{1} \frac{\partial T_{1}}{\partial z}-h_{1}\left[T_{1}-y_{1}(t)\right]=0 \quad \text { at } z=0, \\
\lambda_{n} \frac{\partial T_{n}}{\partial z}+h_{2}(t)\left[T_{n}-y_{2}(t)\right]=0 \quad \text { at } z=H .
\end{gathered}
$$

At the internal boundaries, the slab is subject to the following interface conditions:

$$
\lambda_{i} \frac{\partial T_{i}}{\partial z}+r_{i}\left(T_{i}-T_{i+1}\right)=0
$$

$$
\text { at } z=z_{i} \text { for } i=1,2, \ldots, n-1 \text {, }
$$




$$
\begin{aligned}
\lambda_{i} \frac{\partial T_{i}}{\partial z}= & \lambda_{i+1} \frac{\partial T_{i+1}}{\partial z} \\
& \text { at } z=z_{i} \text { for } i=1,2, \ldots, n-1,
\end{aligned}
$$

where (3) implies the discontinuity of temperature at the interfaces and (4) states that the heat flux is continuous at the interfaces. When $r_{i} \rightarrow \infty$, (3) leads to $T_{i}-T_{i+1}=0$, implying the continuity of temperature or perfect thermal contact at the interfaces. For convenience, we nondimensionalise (1) as follows:

$$
\frac{1}{\bar{\kappa}_{i}} \frac{\partial \theta_{i}}{\partial \tau}=\frac{\partial^{2} \theta_{i}}{\partial \xi^{2}}+\bar{Q}_{i}(\xi, \tau) \quad \text { for } i=1,2, \ldots, n
$$

and the initial, boundary, and interface conditions may be stated as

$$
\begin{gathered}
\theta_{i}=\theta_{\text {in }}(\xi) \\
\text { at } \tau=0 \text { for } i=1,2, \ldots, n, \\
\frac{\partial \theta_{1}}{\partial \xi}-B_{1}\left[\theta_{1}-Y_{1}(\tau)\right]=0 \quad \text { at } \xi=0, \\
\frac{\partial \theta_{n}}{\partial \xi}+B_{2}(\tau) \theta_{n}=\psi(\tau) \quad \text { at } \xi=1, \\
\frac{\partial \theta_{i}}{\partial \xi}+R_{i}\left(\theta_{i}-\theta_{i+1}\right)=0 \\
\text { at } \xi=\xi_{i} \text { for } i=1,2, \ldots, n-1, \\
\bar{\lambda}_{i} \frac{\partial \theta_{i}}{\partial \xi}=\bar{\lambda}_{i+1} \frac{\partial \theta_{i+1}}{\partial \xi} \\
\text { at } \xi=\xi_{i} \text { for } i=1,2, \ldots, n-1,
\end{gathered}
$$

where the dimensionless quantities are defined as follows:

$$
\begin{aligned}
B_{1} & =\frac{h_{1} H}{\lambda_{1}}, \\
B_{2}(\tau) & =\frac{h_{2}(t) H}{\lambda_{n}}, \\
\bar{Q}_{i}(\xi, \tau) & =\frac{Q_{i}(z, t) H^{2}}{\lambda_{i} T_{0}}, \\
R_{i} & =\frac{r_{i} H}{\lambda_{i}}, \\
Y_{j}(\tau) & =\frac{y_{j}(t)}{T_{0}} \text { for } j=1,2, \\
\theta_{i}(\xi, \tau) & =\frac{T_{i}(z, t)}{T_{0}}, \\
\theta_{\text {in }}(\xi) & =\frac{T_{\text {in }}(z)}{T_{0}},
\end{aligned}
$$

$$
\begin{aligned}
\bar{\kappa}_{i} & =\frac{\kappa_{i}}{\kappa_{0}}, \\
\bar{\lambda}_{i} & =\frac{\lambda_{i}}{\lambda_{0}}, \\
\xi & =\frac{z}{H}, \\
\xi_{i} & =\frac{z_{i}}{H}, \\
\tau & =\frac{\kappa_{0} t}{H^{2}}, \\
\psi(\tau) & =B_{2}(\tau) Y_{2}(\tau) .
\end{aligned}
$$

For very large values of $R_{i}$ at the interfacial boundaries, the problem is reduced to that of temperature continuity at the interfaces.

To make the following analysis manageable, $B_{2}(\tau)$ is separated in the form of

$$
B_{2}(\tau)=\delta+F(\tau)
$$

where $\delta$ is the initial value of the Biot number, that is, $B_{2}(0)$. Substituting (12) into (8) yields

$$
\frac{\partial \theta_{n}}{\partial \xi}+\delta \theta_{n}=-F(\tau) \theta_{n}+\psi(\tau) \quad \text { at } \xi=1 .
$$

\section{Analysis}

3.1. Shifting Function Method. To solve the system of (5)-(10), we first introduce the substitution [20]

$$
\theta_{i}(\xi, \tau)=v_{i}(\xi, \tau)+f(\tau) g_{i}(\xi) \quad \text { for } i=1,2, \ldots, n,
$$

where $v_{i}(\xi, \tau)$ is the transformed function, $g_{i}(\xi)$ is a shifting function to be determined, and $f(\tau)$ is the auxiliary function defined as

$$
f(\tau)=-F(\tau) \theta_{n}(1, \tau)+\psi(\tau)
$$

Substituting (14) into (5)-(7), (9), (10), and (13) yields

$$
\begin{aligned}
& \frac{1}{\bar{\kappa}_{i}}\left[\frac{\partial v_{i}(\xi, \tau)}{\partial \tau}+\frac{\mathrm{d} f(\tau)}{\mathrm{d} \tau} g_{i}(\xi)\right]=\frac{\partial^{2} v_{i}(\xi, \tau)}{\partial \xi^{2}} \\
& +f(\tau) \frac{\mathrm{d}^{2} g_{i}(\xi)}{\mathrm{d} \xi^{2}}+\bar{Q}_{i}(\xi, \tau) \\
& \text { for } i=1,2, \ldots, n \text {, } \\
& v_{i}(\xi, 0)+f(0) g_{i}(\xi)=\theta_{\text {in }}(\xi) \quad \text { for } i=1,2, \ldots, n, \\
& \frac{\partial v_{1}(0, \tau)}{\partial \xi}+f(\tau) \frac{\mathrm{d} g_{1}(0)}{\mathrm{d} \xi}-B_{1}\left[v_{1}(0, \tau)\right. \\
& \left.+f(\tau) g_{1}(0)-Y_{1}(\tau)\right]=0,
\end{aligned}
$$




$$
\begin{aligned}
& \frac{\partial v_{n}(1, \tau)}{\partial \xi}+f(\tau) \frac{\mathrm{d} g_{n}(1)}{\mathrm{d} \xi}+\delta\left[v_{n}(1, \tau)\right. \\
& \left.\quad+f(\tau) g_{n}(1)\right]=f(\tau), \\
& \frac{\partial v_{i}\left(\xi_{i}, \tau\right)}{\partial \xi}+f(\tau) \frac{\mathrm{d} g_{i}\left(\xi_{i}\right)}{\mathrm{d} \xi}+R_{i}\left[v_{i}\left(\xi_{i}, \tau\right)\right. \\
& \left.\quad+f(\tau) g_{i}\left(\xi_{i}\right)-v_{i+1}\left(\xi_{i}, \tau\right)-f(\tau) g_{i+1}\left(\xi_{i}\right)\right]=0 \\
& \quad \text { for } i=1,2, \ldots, n-1, \\
& \bar{\lambda}_{i}\left[\frac{\partial v_{i}\left(\xi_{i}, \tau\right)}{\partial \xi}+f(\tau) \frac{\mathrm{d} g_{i}\left(\xi_{i}\right)}{\mathrm{d} \xi}\right]=\bar{\lambda}_{i+1}\left[\frac{\partial v_{i+1}\left(\xi_{i}, \tau\right)}{\partial \xi}\right. \\
& \left.\quad+f(\tau) \frac{\mathrm{d} g_{i+1}\left(\xi_{i}\right)}{\mathrm{d} \xi}\right] \quad \text { for } i=1,2, \ldots, n-1 .
\end{aligned}
$$

Following the procedure of the shifting function method, we suitably assume the shifting function $g_{i}(\xi)$ to be a function satisfying the following system of equations:

$$
\begin{aligned}
& \frac{\mathrm{d}^{2} g_{i}(\xi)}{\mathrm{d} \xi^{2}}=a \\
& \text { for } i=1,2, \ldots, n, \\
& \frac{\mathrm{d} g_{1}(0)}{\mathrm{d} \xi}-B_{1} g_{1}(0)=0, \\
& \frac{\mathrm{d} g_{n}(1)}{\mathrm{d} \xi}=1, \\
& g_{n}(1)=0, \\
& \frac{\mathrm{d} g_{i}\left(\xi_{i}\right)}{\mathrm{d} \xi}+R_{i}\left[g_{i}\left(\xi_{i}\right)-g_{i+1}\left(\xi_{i}\right)\right]=0 \\
& \text { for } i=1,2, \ldots, n-1, \\
& \bar{\lambda}_{i} \frac{\mathrm{d} g_{i}\left(\xi_{i}\right)}{\mathrm{d} \xi}=\bar{\lambda}_{i+1} \frac{\mathrm{d} g_{i+1}\left(\xi_{i}\right)}{\mathrm{d} \xi} \\
& \text { for } i=1,2, \ldots, n-1,
\end{aligned}
$$

where $a$ is an unknown constant. The general solution of (22) is given by

$$
g_{i}(\xi)=\frac{a}{2} \xi^{2}+b_{i} \xi+c_{i}
$$

where $b_{i}$ and $c_{i}(i=1,2, \ldots, n)$ are additional unknown constants. A total of $(2 n+1)$ unknown constants are readily determined from (23)-(27).

Substituting (25) into (14) yields

$$
\theta_{n}(1, \tau)=v_{n}(1, \tau) .
$$

The substitution of (22) and (25) into (16) results in the following partial differential equation:

$$
\begin{gathered}
\frac{\partial v_{i}(\xi, \tau)}{\partial \tau}-\bar{\kappa}_{i} \frac{\partial^{2} v_{i}(\xi, \tau)}{\partial \xi^{2}}-g_{i}(\xi) F(\tau) \dot{v}_{n}(1, \tau) \\
+\left[-g_{i}(\xi) \dot{F}(\tau)+\bar{\kappa}_{i} a F(\tau)\right] v_{n}(1, \tau)
\end{gathered}
$$

$$
=-g_{i}(\xi) \dot{\psi}(\tau)+\bar{\kappa}_{i} a \psi(\tau)+\bar{\kappa}_{i} \bar{Q}_{i}(\xi, \tau)
$$

$$
\text { for } i=1,2, \ldots, n \text {, }
$$

where the "over-dot" denotes the time derivative. Considering (23)-(27), the associated initial, boundary, and interface conditions are obtained as follows:

$$
\begin{aligned}
& v_{i}(\xi, 0)=\theta_{\text {in }}(\xi)-\psi(0) g_{i}(\xi) \quad \text { for } i=1,2, \ldots, n, \\
& \frac{\partial v_{1}(0, \tau)}{\partial \xi}-B_{1}\left[v_{1}(0, \tau)-Y_{1}(\tau)\right]=0, \\
& \frac{\partial v_{n}(1, \tau)}{\partial \xi}+\delta v_{n}(1, \tau)=0, \\
& \frac{\partial v_{i}\left(\xi_{i}, \tau\right)}{\partial \xi}+R_{i}\left[v_{i}\left(\xi_{i}, \tau\right)-v_{i+1}\left(\xi_{i}, \tau\right)\right]=0 \\
& \quad \text { for } i=1,2, \ldots, n-1, \\
& \bar{\lambda}_{i} \frac{\partial v_{i}\left(\xi_{i}, \tau\right)}{\partial \xi}=\bar{\lambda}_{i+1} \frac{\partial v_{i+1}\left(\xi_{i}, \tau\right)}{\partial \xi} \\
& \text { for } i=1,2, \ldots, n-1 .
\end{aligned}
$$

3.2. Orthogonal Expansion Technique. The next step is to find a solution to the multiregion heat conduction problem expressed by (30)-(35). In the literature, various analytical methods are available for transient heat conduction in composite media, such as the orthogonal expansion technique, Laplace transform method, method of separation of variables, Green's function method, and finite integral transform technique. In the present work, Vodicka's method [25], which has been proven effective in analysing multiregion problems with general space- and time-dependent heat sources and nonhomogeneous boundary conditions, is applied to solve the system of (30)-(35).

The solution to (30)-(35) is assumed to be

$$
v_{i}(\xi, \tau)=\sum_{m=1}^{\infty} \phi_{m}(\tau) X_{i m}(\xi)-L_{i}(\xi) Y_{1}(\tau)
$$

$$
\text { for } i=1,2, \ldots, n \text {, }
$$

with the following function:

$$
L_{i}(\xi)=C_{i} \xi+D_{i} \quad \text { for } i=1,2, \ldots, n .
$$

The constants $C_{i}$ and $D_{i}$ are obtained from the following relationships:

$$
\begin{aligned}
\frac{\mathrm{d} L_{1}(0)}{\mathrm{d} \xi}-B_{1} L_{1}(0) & =B_{1}, \\
\frac{\mathrm{d} L_{n}(1)}{\mathrm{d} \xi}+\delta L_{n}(1) & =0, \\
\frac{\mathrm{d} L_{i}\left(\xi_{i}\right)}{\mathrm{d} \xi}+R_{i}\left[L_{i}\left(\xi_{i}\right)-L_{i+1}\left(\xi_{i}\right)\right] & =0,
\end{aligned}
$$




$$
\begin{aligned}
& \bar{\lambda}_{i} \frac{\mathrm{d} L_{i}\left(\xi_{i}\right)}{\mathrm{d} \xi}=\bar{\lambda}_{i+1} \frac{\mathrm{d} L_{i+1}\left(\xi_{i}\right)}{\mathrm{d} \xi}, \\
& \text { for } i=1,2, \ldots, n-1 .
\end{aligned}
$$

The function $X_{i m}(\xi)$ is the solution to the eigenvalue problem corresponding to (30)-(35) and is given as follows:

$$
X_{i m}(\xi)=A_{i m} \cos \left(d_{i m} \xi\right)+B_{i m} \sin \left(d_{i m} \xi\right),
$$

where $d_{i m}=\gamma_{m} /{\sqrt{\bar{\kappa}_{i}}}$ and $\gamma_{m}$ is an eigenvalue. The unknown constants $A_{i m}$ and $B_{i m}$ are determined from the following conditions, which are obtained by substituting (36)-(39) into the boundary and interface conditions, (32)-(35):

$$
\begin{aligned}
& \frac{\mathrm{d} X_{1 m}(0)}{\mathrm{d} \xi}-B_{1} X_{1 m}(0)=0 \\
& \frac{\mathrm{d} X_{n m}(1)}{\mathrm{d} \xi}+\delta X_{n m}(1)=0 \\
& \frac{\mathrm{d} X_{i m}\left(\xi_{i}\right)}{\mathrm{d} \xi}+R_{i}\left[X_{i m}\left(\xi_{i}\right)-X_{(i+1) m}\left(\xi_{i}\right)\right]=0, \\
& \bar{\lambda}_{i} \frac{\mathrm{d} X_{i m}\left(\xi_{i}\right)}{\mathrm{d} \xi}=\bar{\lambda}_{i+1} \frac{\mathrm{d} X_{(i+1) m}\left(\xi_{i}\right)}{\mathrm{d} \xi}, \quad \text { for } i=1,2, \ldots, n-1 .
\end{aligned}
$$

In other words, a system of $2 n$ linear homogeneous equations is obtained for the constants $A_{i m}$ and $B_{i m}$. The eigenvalues $\gamma_{m}(m=1,2, \ldots)$ are obtained from the condition under which this system of equations has a nontrivial solution and are therefore positive roots of the following transcendental equation in matrix notation:

$$
\mathbf{G} \cdot \mathbf{E}_{1} \cdot \mathbf{E}_{2} \cdots \mathbf{E}_{n-1} \cdot \mathbf{u}=0
$$

where

$$
\begin{aligned}
& \mathbf{G}=\left[\begin{array}{ll}
-B_{1} & d_{1 m}
\end{array}\right], \\
& \mathbf{E}_{i}=\mathbf{J}_{i} \cdot \mathbf{K}_{i+1}, \\
& \mathbf{J}_{i}=\left[\begin{array}{ll}
\bar{\lambda}_{i} d_{i m} \cos \left(d_{i m} \xi_{i}\right) & -d_{i m} \cos \left(d_{i m} \xi_{i}\right)-R_{i} \sin \left(d_{i m} \xi_{i}\right) \\
\bar{\lambda}_{i} d_{i m} \sin \left(d_{i m} \xi_{i}\right) & -d_{i m} \sin \left(d_{i m} \xi_{i}\right)+R_{i} \cos \left(d_{i m} \xi_{i}\right)
\end{array}\right], \\
& \mathbf{K}_{i+1} \\
& =\left[\begin{array}{cc}
R_{i} \cos \left[d_{(i+1) m} \xi_{i}\right] & R_{i} \sin \left[d_{(i+1) m} \xi_{i}\right] \\
-\bar{\lambda}_{i+1} d_{(i+1) m} \sin \left[d_{(i+1) m} \xi_{i}\right] & \bar{\lambda}_{i+1} d_{(i+1) m} \cos \left[d_{(i+1) m} \xi_{i}\right]
\end{array}\right], \\
& \mathbf{u}=\left[\begin{array}{l}
d_{n m} \cos \left(d_{n m}\right)+\delta \sin \left(d_{n m}\right) \\
d_{n m} \sin \left(d_{n m}\right)-\delta \cos \left(d_{n m}\right)
\end{array}\right] .
\end{aligned}
$$

By substituting (36) into (31), the following equation is obtained:

$$
\begin{aligned}
W_{i}(\xi) & =\sum_{m=1}^{\infty} \phi_{m}(0) X_{i m}(\xi) \\
& =\theta_{\text {in }}(\xi)-\psi(0) g_{i}(\xi)+L_{i}(\xi) Y_{1}(0)
\end{aligned}
$$

The eigenfunction $X_{i m}(\xi)$ given by (39) has an orthogonal relationship with discontinuous weighting function; it is expressed as follows [26]:

$$
\sum_{i=1}^{n} \frac{\bar{\lambda}_{i}}{\bar{\kappa}_{i}} \int_{\xi_{i-1}}^{\xi_{i}} X_{i l}(\xi) X_{i k}(\xi) \mathrm{d} \xi= \begin{cases}\text { const. } & (l=k) \\ 0 & (l \neq k) .\end{cases}
$$

Functions $g_{i}(\xi), W_{i}(\xi), L_{i}(\xi)$, and $\bar{\kappa}_{i} \bar{Q}_{i}(\xi, \tau)$ and a constant $\bar{\kappa}_{i}$ can be expanded in an infinite series of $X_{i m}(\xi)$ :

$$
\begin{gathered}
g_{i}(\xi)=\sum_{m=1}^{\infty} \alpha_{m} X_{i m}(\xi), \\
W_{i}(\xi)=\sum_{m=1}^{\infty} w_{m} X_{i m}(\xi), \\
L_{i}(\xi)=\sum_{m=1}^{\infty} l_{m} X_{i m}(\xi), \\
\bar{\kappa}_{i} \bar{Q}_{i}(\xi, \tau)=\sum_{m=1}^{\infty} q_{m}(\tau) X_{i m}(\xi), \\
\bar{\kappa}_{i}=\sum_{m=1}^{\infty} \eta_{m} X_{i m}(\xi),
\end{gathered}
$$

where the expansion coefficients are given by

$$
\begin{gathered}
\alpha_{m}=\frac{1}{N_{m}} \sum_{i=1}^{n} \frac{\bar{\lambda}_{i}}{\bar{\kappa}_{i}} \int_{\xi_{i-1}}^{\xi_{i}} g_{i}(\xi) X_{i m}(\xi) \mathrm{d} \xi \\
w_{m}=\frac{1}{N_{m}} \sum_{i=1}^{n} \frac{\bar{\lambda}_{i}}{\overline{\bar{\kappa}}_{i}} \int_{\xi_{i-1}}^{\xi_{i}} W_{i}(\xi) X_{i m}(\xi) \mathrm{d} \xi \\
l_{m}=\frac{1}{N_{m}} \sum_{i=1}^{n} \frac{\bar{\lambda}_{i}}{\bar{\kappa}_{i}} \int_{\xi_{i-1}}^{\xi_{i}} L_{i}(\xi) X_{i m}(\xi) \mathrm{d} \xi
\end{gathered}
$$

$$
\begin{aligned}
q_{m}(\tau) & =\frac{1}{N_{m}} \sum_{i=1}^{n} \bar{\lambda}_{i} \int_{\xi_{i-1}}^{\xi_{i}} \bar{Q}_{i}(\xi, \tau) X_{i m}(\xi) \mathrm{d} \xi \\
\eta_{m} & =\frac{1}{N_{m}} \sum_{i=1}^{n} \bar{\lambda}_{i} \int_{\xi_{i-1}}^{\xi_{i}} X_{i m}(\xi) \mathrm{d} \xi
\end{aligned}
$$

in which the norm $N_{m}$ is

$$
N_{m}=\sum_{i=1}^{n} \frac{\bar{\lambda}_{i}}{\bar{\kappa}_{i}} \int_{\xi_{i-1}}^{\xi_{i}}\left[X_{i m}(\xi)\right]^{2} \mathrm{~d} \xi .
$$

The substitution of (36) and (45) into (30) yields the following equation:

$$
\begin{aligned}
& \sum_{m=1}^{\infty}\left\{\dot{\phi}_{m}(\tau) X_{i m}(\xi)-l_{m} \dot{Y}_{1}(\tau) X_{i m}(\xi)\right. \\
& \quad+\gamma_{m}^{2} \phi_{m}(\tau) X_{i m}(\xi)-g_{i}(\xi) F(\tau) \dot{\phi}_{m}(\tau) X_{n m}(1) \\
& \quad+\left[-g_{i}(\xi) \dot{F}(\tau)+a F(\tau)\right] \phi_{m}(\tau) X_{n m}(1) \\
& \quad+\alpha_{m} L_{n}(1) X_{i m}(\xi)\left[F(\tau) \dot{Y}_{1}(\tau)+\dot{F}(\tau) Y_{1}(\tau)\right]
\end{aligned}
$$




$$
\begin{aligned}
& -a F(\tau) L_{n}(1) Y_{1}(\tau) \eta_{m} X_{i m}(\xi)+\alpha_{m} \dot{\psi}(\tau) X_{i m}(\xi) \\
& \left.-a \psi(\tau) \eta_{m} X_{i m}(\xi)-q_{m}(\tau) X_{i m}(\xi)\right\}=0
\end{aligned}
$$

Equation (48) is satisfied if the expression in the braces is zero; that is,

$$
\begin{aligned}
\dot{\phi}_{m}(\tau) X_{i m}(\xi)+\gamma_{m}^{2} \phi_{m}(\tau) X_{i m}(\xi) \\
\quad-g_{i}(\xi) F(\tau) \dot{\phi}_{m}(\tau) X_{n m}(1) \\
\quad+\left[-g_{i}(\xi) \dot{F}(\tau)+a F(\tau)\right] \phi_{m}(\tau) X_{n m}(1) \\
=l_{m} \dot{Y}_{1}(\tau) X_{i m}(\xi) \\
\quad-\alpha_{m} L_{n}(1) X_{i m}(\xi)\left[F(\tau) \dot{Y}_{1}(\tau)+\dot{F}(\tau) Y_{1}(\tau)\right] \\
\quad+a F(\tau) L_{n}(1) Y_{1}(\tau) \eta_{m} X_{i m}(\xi)
\end{aligned}
$$

$$
\begin{aligned}
& -\alpha_{m} \dot{\psi}(\tau) X_{i m}(\xi)+a \psi(\tau) \eta_{m} X_{i m}(\xi) \\
& +q_{m}(\tau) X_{i m}(\xi) .
\end{aligned}
$$

Equation (49) is multiplied by $\left(\bar{\lambda}_{i} / \bar{\kappa}_{i}\right) X_{i m}(\xi)$, integrated over the region $\left[\xi_{i-1}, \xi_{i}\right]$, and then summed up for $i=1,2, \ldots, n$ to yield

$$
\dot{\phi}_{m}(\tau)+\frac{\gamma_{m}^{2}-\beta_{m} \dot{F}(\tau)+a \chi_{m} F(\tau)}{1-\beta_{m} F(\tau)} \phi_{m}(\tau)=\varsigma_{m}(\tau),
$$

where $\beta_{m}$ and $\chi_{m}$ are given by

$$
\begin{aligned}
& \beta_{m}=X_{n m}(1) \cdot \alpha_{m} \\
& \chi_{m}=X_{n m}(1) \cdot \eta_{m}
\end{aligned}
$$

and $\zeta_{m}(\tau)$ is defined as

$$
\varsigma_{m}(\tau)=\frac{\dot{Y}_{1}(\tau)\left[l_{m}-F(\tau) L_{n}(1) \alpha_{m}\right]+L_{n}(1) Y_{1}(\tau)\left[a F(\tau) \eta_{m}-\alpha_{m} \dot{F}(\tau)\right]-\dot{\psi}(\tau) \alpha_{m}+a \psi(\tau) \eta_{m}+q_{m}(\tau)}{1-\beta_{m} F(\tau)}
$$

By solving (50) with the condition $\phi_{m}(0)=w_{m}$, which is obtained from the comparison between (43) and $(45)_{2}$, we obtain $\phi_{m}(\tau)$ as

$$
\begin{gathered}
\phi_{m}(\tau)=e^{-\int_{0}^{\tau}\left(\left(\gamma_{m}^{2}-\beta_{m} \dot{F}(s)+a \chi_{m} F(s)\right) /\left(1-\beta_{m} F(s)\right)\right) \mathrm{d} s}\left[w_{m}\right. \\
\left.+\int_{0}^{\tau} \zeta_{m}(\mu) \cdot e^{\int_{0}^{\mu}\left(\left(\gamma_{m}^{2}-\beta_{m} \dot{F}(s)+a \chi_{m} F(s)\right) /\left(1-\beta_{m} F(s)\right)\right) \mathrm{d} s} \mathrm{~d} \mu\right] .
\end{gathered}
$$

After substituting (28), (29), (36), (37), and (39) back to (14), the dimensionless temperature in the $i$ th layer $\xi \in$ $\left[\xi_{i-1}, \xi_{i}\right]$ of the composite slab is derived as

$$
\begin{aligned}
& \theta_{i}(\xi, \tau)=\sum_{m=1}^{\infty} \phi_{m}(\tau)\left\{A_{i m} \cos \left(d_{i m} \xi\right)+B_{i m} \sin \left(d_{i m} \xi\right)\right. \\
& -\left(\frac{a}{2} \xi^{2}+b_{i} \xi+c_{i}\right) F(\tau) \\
& \left.\cdot\left[A_{n m} \cos \left(d_{n m}\right)+B_{n m} \sin \left(d_{n m}\right)\right]\right\}-Y_{1}(\tau)\left[C_{i} \xi\right. \\
& \left.+D_{i}-\left(\frac{a}{2} \xi^{2}+b_{i} \xi+c_{i}\right) F(\tau)\left(C_{n}+D_{n}\right)\right]+\left(\frac{a}{2} \xi^{2}\right. \\
& \left.+b_{i} \xi+c_{i}\right) \psi(\tau) .
\end{aligned}
$$

In the methodology for deriving the temperature solution, main differences from the counterpart for single layer [20] are the following: (i) the solution is first shifted in each layer by using not a single shifting function across the total thickness but shifting functions that differ for each layer (see (14)); (ii) since there exist interfaces, the interface conditions (see (20) and (21)) are added; and (iii) various functions are expanded by using eigenfunctions that fulfil an orthogonal relationship with discontinuous weighting function (see (44)).

\section{Verification of Analytical Solution}

To illustrate the applicability of the solution derived, first, a single-layered heat conduction problem, whose solution was previously known [20], is modelled as a multilayer problem, with adjacent layers having identical physical properties. This problem (hereinafter called Case A) involves a timedependent HTC (or Biot number) on one boundary surface of the slab. The following numerical parameters are adopted [20]: $\theta_{\text {in }}=0.336, B_{1}=0, B_{2}(\tau)=1.2-\exp (-2 \tau), Y_{2}(\tau)=1.5-$ $0.5 \exp (-\tau)$, and $\bar{Q}_{i}=0$. For the numerical computation, we use the analytical solution, (see (54)), taking $n=5$ and $\xi_{i}(i=$ $1,2,3,4)$ evenly spaced between 0 and 1 , assuming perfect contact at the interfaces and uniform thermal conductivity and diffusivity within the entire region; that is, $R_{i}=\infty$, $\bar{\lambda}_{i}=1$, and $\bar{\kappa}_{i}=1$.

Second, we consider another test case with a constant Biot number to verify our analytical solution for multiregion heat conduction. This test case (called conveniently Case B here) represents transient diffusion in a composite slab consisting of $n=10$ layers with evenly spaced $\xi_{i}$. The material properties are taken as $\bar{\lambda}_{i}=\bar{\kappa}_{i}=1$ for $i=1,3,5,7,9$ and $\bar{\lambda}_{i}=\bar{\kappa}_{i}=0.1$ for $i=2,4,6,8,10$ [27]. The constants appearing in the initial and boundary conditions are $\theta_{\text {in }}=0, B_{1}=\infty, B_{2}=0$, and $Y_{1}=1$. Moreover, imperfect thermal contact is applied at the interfaces $\left(R_{i}=0.5 / \bar{\kappa}_{i}, i=1,2, \ldots, 9\right)$.

Figures 2 and 3 plot the analytical solution (see (54)) and numerical results previously published for Cases $\mathrm{A}$ and $\mathrm{B}$, respectively. The derived analytical solution is computed using the first 20 terms in the infinite series. In both cases, the 


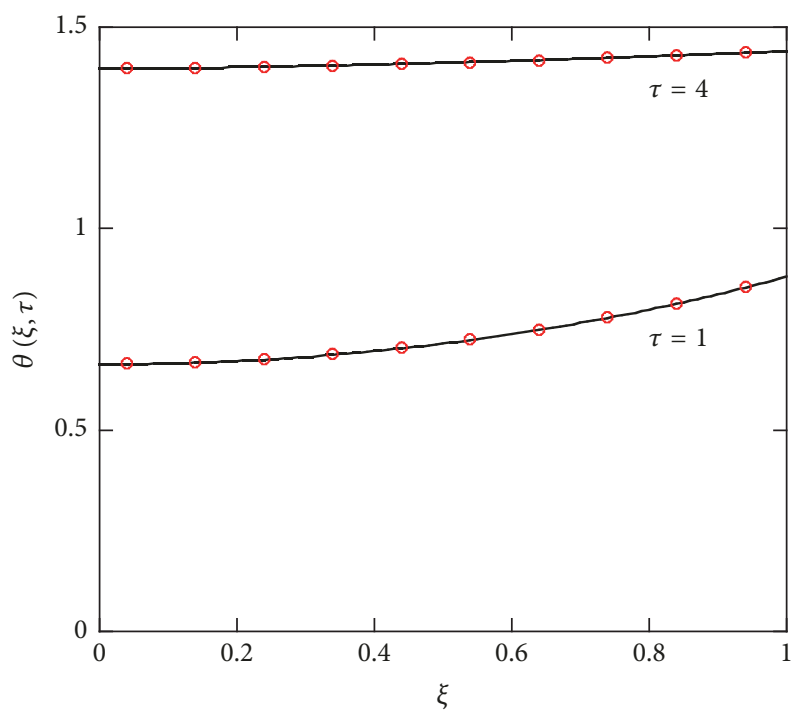

FIgURE 2: Solution verification for Case A: the derived analytical solution is plotted as a solid line and the solution presented in [20] is plotted as circles.

analytical solution is in good agreement with the solutions of the existing literature. Tables 1 and 2 show the relative error defined by

$$
\text { error }=\frac{\max _{i=1,2, \ldots, n}\left|\theta_{i}\left(\xi_{i}, \tau\right)-\Theta\left(\xi_{i}, \tau\right)\right|}{\max _{i=1,2, \ldots, n}\left|\theta_{i}\left(\xi_{i}, \tau\right)\right|}
$$

where $\theta_{i}$ is the derived solution and $\Theta$ is the solution obtained using the analytical method [20] for Case A and one of either the semianalytical method [27] or Fokas method [28] for Case B. As shown in the tables, the relative error is within approximately $0.2 \%$.

\section{Illustrative Examples}

In this section, we apply the analytical solution described in Section 3 to two industrial applications. Because we focus on the effect of time-dependent HTC at the heated surface on the transient temperature distribution of composite media, only the cases with constant ambient temperature and no internal heat generation are considered here.

5.1. Bilayered Plate with Exponentially Varying HTC. Consider the case of a bilayered plate of aluminium and lead, whose initial temperature is zero. Equal thicknesses for both layers are used, which leads to $\xi_{1}=1 / 2$, with perfect thermal contact. For $\tau>0$, the surrounding medium on the side of lead is raised to a temperature of $Y_{2}=1$, while the other surface of the plate is insulated $\left(B_{1}=0\right)$. For the Biot number at $\xi=$ 1 , we choose $B_{2}(\tau)=p_{1}-p_{2} \exp (-s \tau)$, where $p_{1}, p_{2}$, and $s$ are arbitrary constants. The following material properties have been used in the calculations [26]: $\bar{\lambda}_{1}=5.850, \bar{\lambda}_{2}=1$, $\bar{\kappa}_{1}=4.096$, and $\bar{\kappa}_{2}=1$.

The resulting temperature distributions are given in Figures 4 and 5 for various values of location $\xi$ and Fourier

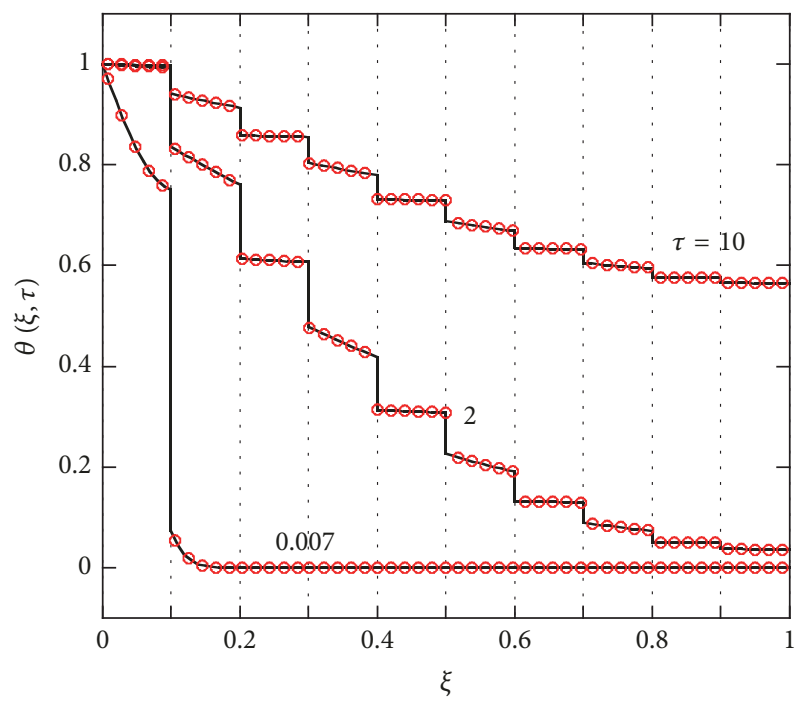

FIgURE 3: Solution verification for Case B: the derived analytical solution is plotted as a solid line and the solution presented in [27] is plotted as circles.

TABLE 1: Relative error in Case A.

\begin{tabular}{lcc}
\hline Fourier number $(\tau)$ & 1 & 4 \\
\hline Error & $2.08 \times 10^{-3}$ & $1.39 \times 10^{-4}$ \\
\hline
\end{tabular}

number $\tau$. In Figure 4, the temporal variation in $B_{2}$ is also shown in the top part, which ranges from 0.2 to 1.2 . In addition, Table 3 lists the temperatures at both the insulated and heated surfaces, for different number of terms in the series, to show the convergence behaviour of the present analytical solution. From Table 3, it can be seen that the convergence of the present solution is very fast. When the Fourier number is larger than 0.2 , the error for the oneterm approximation solution is less than $0.3 \%$. This fast convergence of the solution is one of the notable features that the analytical solutions obtained through the shifting function method have $[18,20]$. Note that all the graphical representations shown in Section 5 are based on the results obtained from the four-term approximation.

As shown in Figure 4, the temporal variations in the temperatures correspond to the variation in $B_{2}$, being the most remarkable for $s=2$, for which the rate of increase in $B_{2}$ is the highest. The time-dependent Biot number $B_{2}$ greatly affects the temperature of the heated surface for a small value of $\tau$, having a lagged influence on the temperature at the insulated boundary. It is worth noting that the influence on the insulated boundary is not negligible. Figure 5 shows that different $s$ values make a measurable difference in the profiles of $\theta$ throughout the thickness.

5.2. Three-Layered Composite with Periodically Varying HTC. Consider the case of a three-layered composite made of tin, aluminium, and lead with interfaces at $\xi_{1}=1 / 3$ and $\xi_{2}=2 / 3$ [26]. Again, we assume perfect thermal contact. The initial and boundary conditions are the same as those in Section 5.1, except that $B_{2}(\tau)=p_{1}-p_{2} \cos (\omega \tau)$, where $\omega$ is a constant. The 


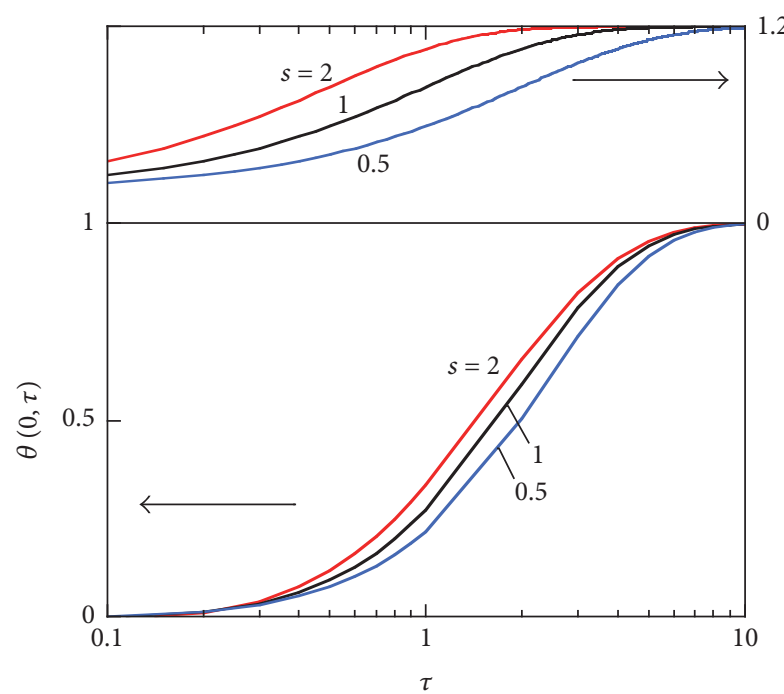

(a)

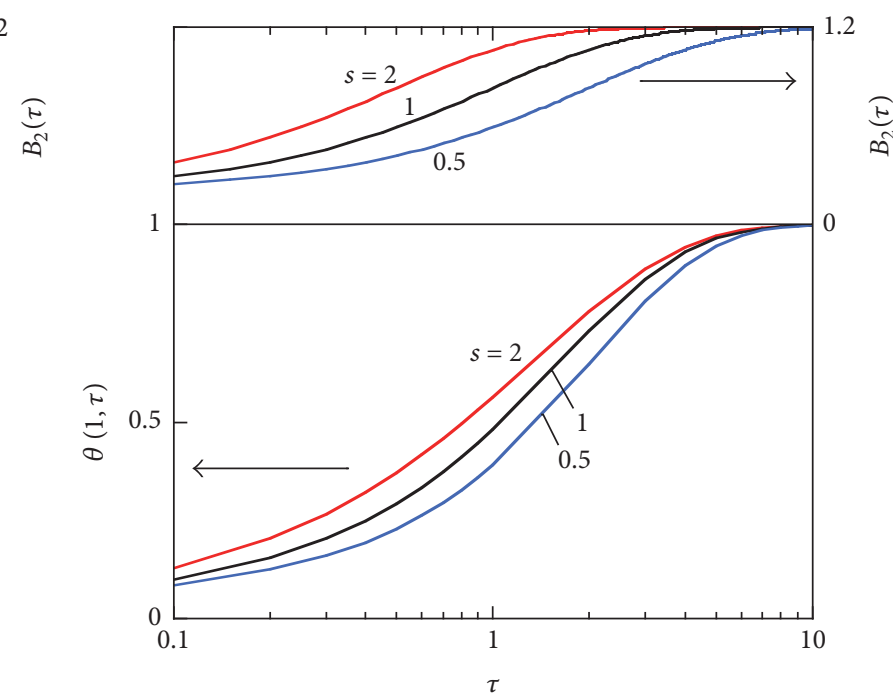

(b)

Figure 4: Influence of parameter $s$ on the temperature variation of the bilayered plate at two locations: (a) $\xi=0$ and (b) $\xi=1$ for $p_{1}=1.2$ and $p_{2}=1$.

TABLE 2: Relative error in Case B.

\begin{tabular}{lccc}
\hline Fourier number $(\tau)$ & 0.007 & 2 & 10 \\
\hline Error (semianalytical) & $1.56 \times 10^{-3}$ & $5.64 \times 10^{-4}$ & $8.62 \times 10^{-4}$ \\
Error (Fokas method) & $5.32 \times 10^{-5}$ & $5.04 \times 10^{-5}$ & $5.01 \times 10^{-5}$ \\
\hline
\end{tabular}

TABLE 3: Dimensionless temperature $\theta$ for different number of terms in the series of (54), for $s=1, p_{1}=1.2$, and $p_{2}=1$.

\begin{tabular}{lcccccccc}
\hline$\tau$ & \multicolumn{3}{c}{$\theta(0, \tau)$} & & \multicolumn{3}{c}{$\theta(1, \tau)$} \\
& 1 term & 2 terms & 4 terms & 10 terms & 1 term & 2 terms & 4 terms & 10 terms \\
\hline 0.1 & -0.0064 & -0.0024 & -0.0024 & -0.0024 & 0.1116 & 0.1006 & 0.1006 \\
0.2 & 0.0110 & 0.0119 & 0.0119 & 0.0119 & 0.1590 & 0.1563 & 0.1563 \\
0.3 & 0.0344 & 0.0345 & 0.0345 & 0.0345 & 0.2051 & 0.2045 & 0.2045 \\
0.4 & 0.0622 & 0.0622 & 0.0622 & 0.0622 & 0.2498 & 0.2497 & 0.2497 \\
0.5 & 0.0934 & 0.0935 & 0.0935 & 0.0935 & 0.2928 & 0.2928 & 0.2928 \\
1 & 0.2723 & 0.2723 & 0.2723 & 0.2723 & 0.4807 & 0.4807 & 0.4807 \\
1.5 & 0.4461 & 0.4461 & 0.4461 & 0.4461 & 0.6241 & 0.6241 & 0.2045 \\
2 & 0.5903 & 0.5903 & 0.5903 & 0.5903 & 0.7300 & 0.7300 & 0.2497 \\
3 & 0.7853 & 0.7853 & 0.7853 & 0.7853 & 0.8624 & 0.8624 & 0.4807 \\
\hline
\end{tabular}

following thermal conductivities and diffusivities have been used [26]: $\bar{\lambda}_{1}=1.900, \bar{\lambda}_{2}=5.850, \bar{\lambda}_{3}=1, \bar{\kappa}_{1}=1.633, \bar{\kappa}_{2}=$ 4.096, and $\bar{\kappa}_{3}=1$.

Figure 6 illustrates the temperature variations at two external boundaries for different $\omega$ values. The periodically varying $B_{2}$ ranges from 0.2 to 2.2 , as shown in the top part of the figures. Visible oscillations are observed in both temperatures for $\omega=2$ and 4 , for which the change cycle of $B_{2}$ is short. Moreover, unlike the case in Section 5.1, there are time slots during which the magnitude relation of the Biot numbers is not necessarily consistent with that of the temperatures for different $\omega$ values. The temperature profiles of the composite at $\tau=1$ and 4 are plotted in Figure 7, which are all monotonic functions of $\xi$ with discontinuous gradient at the interfaces. It can be observed that the effects of the temporal variations in $B_{2}$ propagate through the whole thickness of the three-layered composite.

\section{Conclusions}

An analytical method based on the shifting function method has been presented for solving the one-dimensional transient heat conduction problem with time-dependent HTC for a composite slab consisting of an arbitrary number of layers. Applying the shifting function method yields new partial differential equations under conventional types of initial, boundary, and interfacial conditions. In this paper, the derived equations were solved by using an orthogonal expansion based on the Vodicka type of orthogonality relationship. Numerical calculations were performed for two 


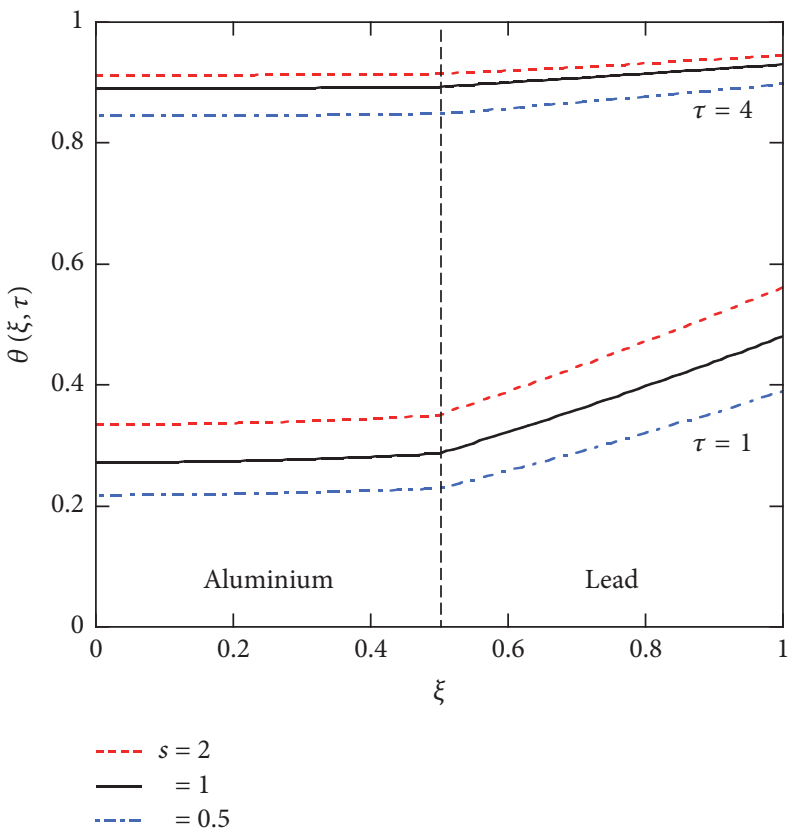

FIGURE 5: Temperature distributions along the thickness of the bilayered plate at $\tau=1$ and 4 with different $s$ values for $p_{1}=1.2$ and $p_{2}=1$.

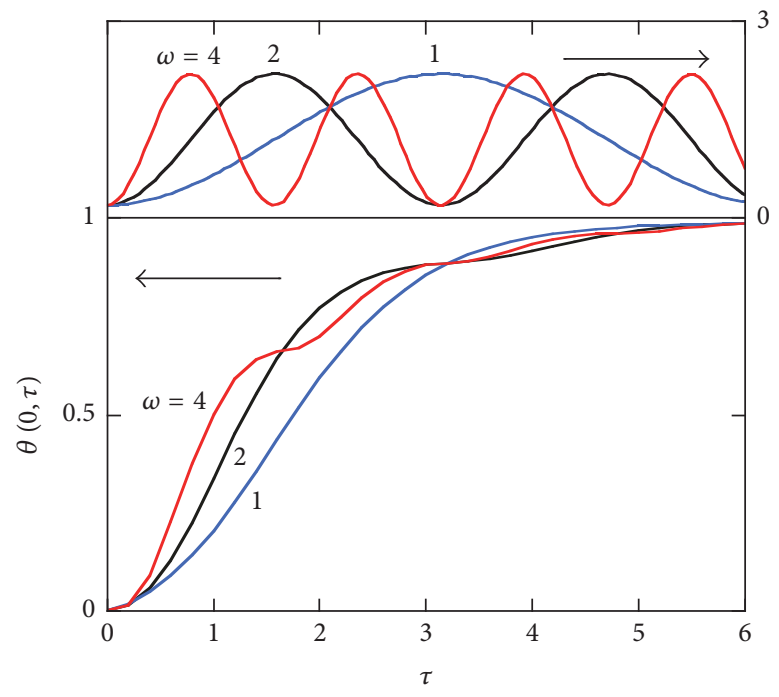

(a)

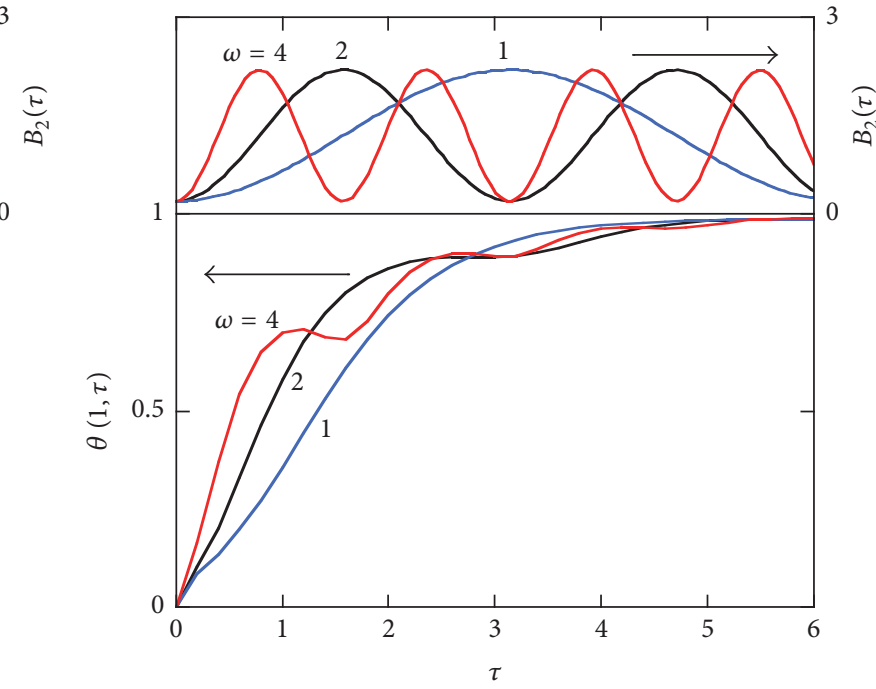

(b)

FiguRE 6: Influence of parameter $\omega$ on the temperature variation of the three-layered composite at two locations: (a) $\xi=0$ and (b) $\xi=1$ for $p_{1}=1.2$ and $p_{2}=1$.

representative composite slabs. The numerical results demonstrated the effects of temporal variations in the HTC on the transient temperature field, in particular, on the temperatures at two external boundaries.

This paper considers the temporal variation in the HTC at only one external boundary. The solution needs to be extended so that the time-dependency of the HTCs at both boundaries can be considered. This might possibly be achieved by introducing two different shifting functions [19, 29]. The present analytical method can easily be extended to other simple geometries; it changes nothing beyond the forms of the eigenfunction, shifting function, function $L_{i}(\xi)$, and the orthogonal relationship of (44). The analytical solution derived is applicable to nonhomogeneous materials, such as functionally graded materials, under the laminate approximation theory [30].

The presented analytical method is available for transient heat conduction problems with arbitrary time-dependent HTC. However, currently, it cannot be applied to those in which the HTC is a function of position or of temperature. 




Figure 7: Temperature distributions along the thickness of the three-layered composite at $\tau=1$ and 4 with different $\omega$ values for $p_{1}=1.2$ and $p_{2}=1$.

\section{Nomenclature}

$\begin{array}{ll}a: & \text { Constant in } g_{i}(\xi) \\ A_{i m}, B_{i m}: & \text { Constants in } X_{i m}(\xi) \\ B_{1}, B_{2}(\tau): & \text { Biot numbers } \\ b_{i}, c_{i}: & \text { Constants in } g_{i}(\xi) \\ C_{i}, D_{i}: & \text { Constants in } L_{i}(\xi) \\ d_{i m}: & \text { Constant }\left(=\gamma_{m} / \sqrt{\bar{\kappa}_{i}}\right) \\ f(\tau): & \text { Auxiliary time function, defined by }(15) \\ F(\tau): & \text { Nonstationary part of } B_{2}(\tau) \\ g_{i}(\xi): & \text { Shifting function, defined by }(14) \\ h_{1}, h h_{2}(t): & \text { Heat transfer coefficients } \\ H: & \text { Thickness } \\ i: & \text { Positive integer } \\ l_{m}: & \text { Expansion coefficient, defined by }(46) \\ L_{i}(\xi): & \text { Function of } \xi, \text { defined by }(36) \\ m_{,} n: & \text { Integers } \\ N_{m}: & \text { Norm, defined by }(47) \\ p_{1}, p_{2}: & \text { Constants in } B_{2}(\tau) \\ q_{m}(\tau): & \text { Function of time, defined by }(46) \\ Q_{i}(\xi, \tau): & \text { Distributed source in the } i \text { th region } \\ r_{i}: & \text { Conductance coefficient between } i \text { th } \\ R_{i}: & \text { and }(i+1) \text { th regions } \\ s: & \text { Dimensionless conductance coefficient } \\ t: & \text { Constant } \\ T_{i}(x, t): & \text { Time } \\ v_{i}(\xi, \tau): & \text { Temperature in the } i \text { th region } \\ w_{m}: & \text { Expansion coefficient, defined by }(46) \\ W_{i}(\xi): & \text { Derived function of } \xi, \text { defined by }(43) \\ X_{i m}(\xi): & \text { Eigenfunction } \\ y_{1}(t), y_{2}(t): & \text { Temperatures of surroundings } \\ & \end{array}$

$$
\begin{array}{ll}
Y_{1}(\tau), Y_{2}(\tau): & \begin{array}{l}
\text { Dimensionless temperatures of } \\
\text { surroundings }
\end{array} \\
z: & \text { Spatial coordinate. }
\end{array}
$$

Greek Symbols

$\begin{array}{ll}\alpha_{m}: & \text { Expansion coefficient, defined by }(46) \\ \beta_{m}: & \text { Constant }\left(=X_{n m}(1) \cdot \alpha_{m}\right) \\ \chi_{m}: & \text { Constant }\left(=X_{n m}(1) \cdot \eta_{m}\right) \\ \delta: & \text { Initial value of } B_{2}(\tau) \\ \phi_{m}(\tau): & \text { Function of time, defined by }(36) \\ \gamma_{m}: & \text { Eigenvalue } \\ \eta_{m}: & \text { Expansion coefficient, defined by }(46) \\ \psi(\tau): & \text { Function of time }\left(=B_{2}(\tau) \cdot Y_{2}(\tau)\right) \\ \kappa_{i}: & \text { Thermal diffusivity in the } i \text { th region } \\ \bar{\kappa}_{i}: & \text { Ratio of thermal diffusivities } \\ \lambda_{i}: & \text { Thermal conductivity in the } i \text { th region } \\ \bar{\lambda}_{i}: & \text { Ratio of thermal conductivities } \\ \theta_{i}(\xi, \tau): & \text { Dimensionless temperature in the } i \text { th region } \\ \tau: & \text { Fourier number } \\ \omega: & \text { Constant } \\ \zeta_{m}(\tau): & \text { Function of time, defined by }(52) \\ \xi: & \text { Dimensionless spatial coordinate. }\end{array}$

\section{Subscripts}

in: Initial value

0: Reference value.

\section{Conflicts of Interest}

The author declares that there are no conflicts of interest regarding the publication of this article.

\section{Acknowledgments}

The author would like to thank Drs. N. E. Sheils, T. W. Tu, and E. J. Carr for technical assistance in verifying the analytical solution.

\section{References}

[1] M. Mori and M. Kondo, "Temperature and Thermal Stress Analysis in a Structure with Uncertain Heat Transfer Boundary Conditions," Transactions of the Japan Society of Mechanical Engineers Series A, vol. 59, no. 562, pp. 1514-1518, 1993.

[2] J. J. Thompson and Z. J. Holy, "Axisymmetric thermal response problems for a spherical fuel element with time dependent heat transfer coefficients," Nuclear Engineering and Design, vol. 9, no. 1, pp. 29-44, 1969.

[3] T. G. Kim and Z. H. Lee, "Time-varying heat transfer coefficients between tube-shaped casting and metal mold," International Journal of Heat and Mass Transfer, vol. 40, no. 15, pp. 35133525, 1997.

[4] S. S. Sazhin, P. A. Krutitskii, W. A. Abdelghaffar et al., "Transient heating of diesel fuel droplets," International Journal of Heat and Mass Transfer, vol. 47, no. 14-16, pp. 3327-3340, 2004.

[5] I. M. Fedotkin, A. M. Aizen, and I. A. Goloshchuk, "Application of integral equations to heat conduction problems in which the 
heat transfer coefficient varies," Journal of Engineering Physics, vol. 28, no. 3, pp. 392-395, 1975.

[6] B. Nicolai and J. De Baerdemaeker, "Simulation of heat transfer in foods with stochastic initial and boundary conditions," Trans IChemE 70C, pp. 78-82, 1992.

[7] B. Y. Lyubov and N. I. Yalovoi, "Heat conductivity of a body with variable heat exchange coefficient," Journal of Engineering Physics, vol. 17, no. 4, pp. 1264-1270, 1972.

[8] N. M. Becker, R. L. Bivins, Y. C. Hsu, H. D. Murphy, A. B. White Jr., and G. M. Wing, "Heat diffusion with timedependent convective boundary condition," International Journal for Numerical Methods in Engineering, vol. 19, no. 12, pp. 1871-1880, 1983.

[9] M. B. Abd-el-Malek and M. M. Helal, "Group method solution for solving nonlinear heat diffusion problems," Applied Mathematical Modelling, vol. 30, no. 9, pp. 930-940, 2006.

[10] V. V. Ivanov and V. V. Salomatov, "On the calculation of the temperature field in solids with variable heat-transfer coefficients," Journal of Engineering Physics, vol. 9, no. 1, pp. 63-64, 1967.

[11] V. V. Ivanov and V. V. Salomatov, "Unsteady temperature field in solid bodies with variable heat transfer coefficient," Journal of Engineering Physics, vol. 11, no. 2, pp. 151-152, 1969.

[12] Y. S. Postol'nik, "One-dimensional convective heating with a time-dependent heat-transfer coefficient," Journal of Engineering Physics, vol. 18, no. 2, pp. 233-238, 1972.

[13] V. N. Kozlov, "Temperature field of an infinite plate in the case of a variable heat-exchange coefficient," Journal of Engineering Physics, vol. 16, no. 1, pp. 95-97, 1972.

[14] M. N. Özisik and R. L. Murray, "On the solution of linear diffusion problems with variable boundary condition parameters," Journal of Heat Transfer, vol. 96, no. 1, pp. 48-51, 1974.

[15] M. D. Mikhailov, "On the solution of the heat equation with time dependent coefficient," International Journal of Heat and Mass Transfer, vol. 18, no. 2, pp. 344-345, 1975.

[16] R. J. Moitsheki, “Transient heat diffusion with temperaturedependent conductivity and time-dependent heat transfer coefficient," Mathematical Problems in Engineering, Article ID 347568, 9 pages, 2008.

[17] V. N. Kozlov, "Solution of heat-conduction problem with variable heat-exchange coefficient," Journal of Engineering Physics, vol. 18, no. 1, pp. 100-104, 1972.

[18] H. T. Chen, S. L. Sun, H. C. Huang, and S. Y. Lee, "Analytic closed solution for the heat conduction with time dependent heat convection coefficient at one boundary," Computer Modeling in Engineering \& Sciences, vol. 59, no. 2, pp. 107-126, 2010.

[19] T. W. Tu and S. Y. Lee, "Analytical solution of heat conduction for hollow cylinders with time-dependent boundary condition and time-dependent heat transfer coefficient," Journal of Applied Mathematics, Article ID 203404, 9 pages, 2015.

[20] T. W. Tu and S. Y. Lee, "Exact temperature field in a slab with time varying ambient temperature and time-dependent heat transfer coefficient," International Journal of Thermal Sciences, vol. 116, pp. 82-90, 2017.

[21] O. T. Il'chenko, "Temperature field of a two-layered plate with time-varying heat-transfer conditions," Journal of Engineering Physics, vol. 19, no. 6, pp. 1567-1570, 1973.

[22] I. M. Prikhod'ko, “Thermal conductivity of a two-layer wall for a time-varying heat-transfer coefficient and ambient temperature," Journal of Engineering Physics, vol. 18, no. 2, pp. 239-242, 1972.
[23] Y. Yener and M. N. Ozisik, "On the solution of unsteady heat conduction in multi-region finite media with time dependent heat transfer coefficient," in Proceedings of the 5th International Heat Transfer Conference, Tokyo, pp. 188-192, 1974.

[24] Z. J. Holy, "Temperature and stresses in reactor fuel elements due to time- and space-dependent heat-transfer coefficients," Nuclear Engineering and Design, vol. 18, no. 1, pp. 145-197, 1972.

[25] V. Vodička, "Eindimensionale Wärmeleitung in geschichteten Körpern," Mathematische Nachrichten, vol. 14, pp. 47-55, 1955.

[26] G. P. Mulholland and M. H. Cobble, "Diffusion through composite media," International Journal of Heat and Mass Transfer, vol. 15, no. 1, pp. 147-160, 1972.

[27] E. J. Carr and I. W. Turner, "A semi-analytical solution for multilayer diffusion in a composite medium consisting of a large number of layers," Applied Mathematical Modelling: Simulation and Computation for Engineering and Environmental Systems, vol. 40, no. 15-16, pp. 7034-7050, 2016.

[28] N. E. Sheils, "Multilayer diffusion in a composite medium with imperfect contact," Applied Mathematical Modelling: Simulation and Computation for Engineering and Environmental Systems, vol. 46, pp. 450-464, 2017.

[29] S. Y. Lee and C. C. Huang, "Analytic solutions for heat conduction in functionally graded circular hollow cylinders with time-dependent boundary conditions," Mathematical Problems in Engineering, vol. 2013, Article ID 816385, 8 pages, 2013.

[30] H. M. Wang, "An effective approach for transient thermal analysis in a functionally graded hollow cylinder," International Journal of Heat and Mass Transfer, vol. 67, pp. 499-505, 2013. 


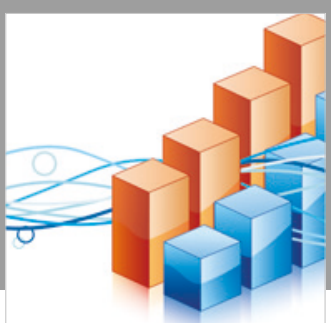

Advances in

Operations Research

\section{-n-m}



Journal of

Applied Mathematics
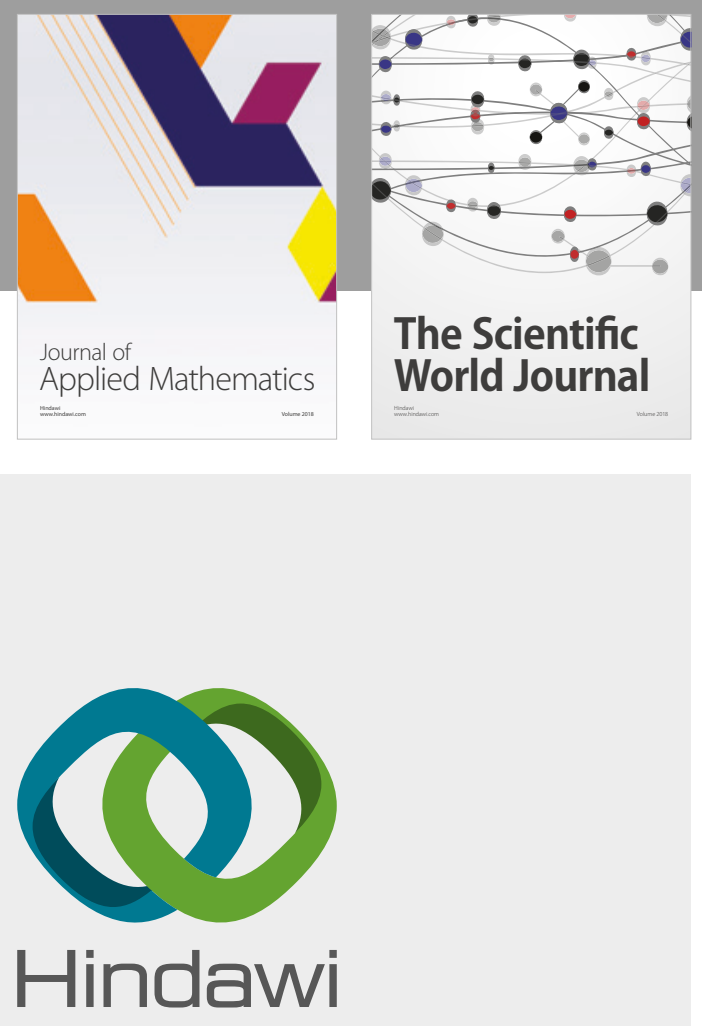

Submit your manuscripts at

www.hindawi.com

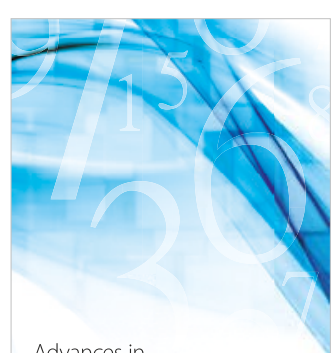

Advances in
Numerical Analysis
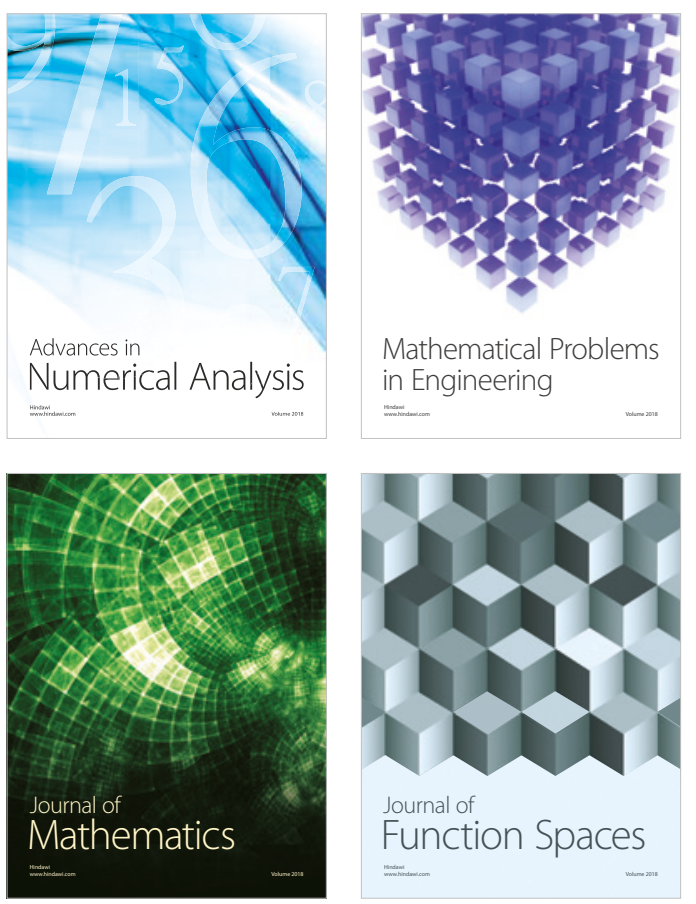

Mathematical Problems in Engineering

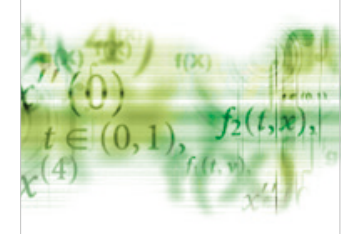

International Journal of

Differential Equations

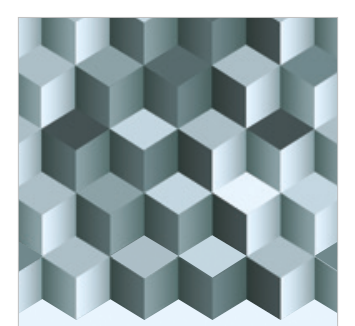

Journal of

Function Spaces

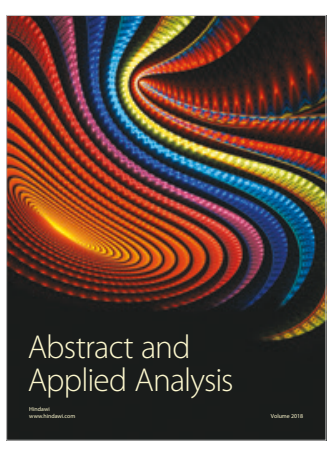

The Scientific

World Journal

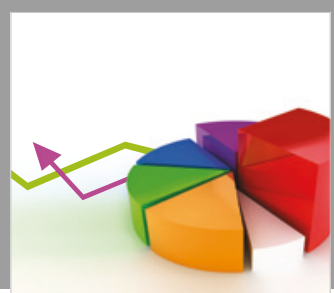

Journal of

Probability and Statistics
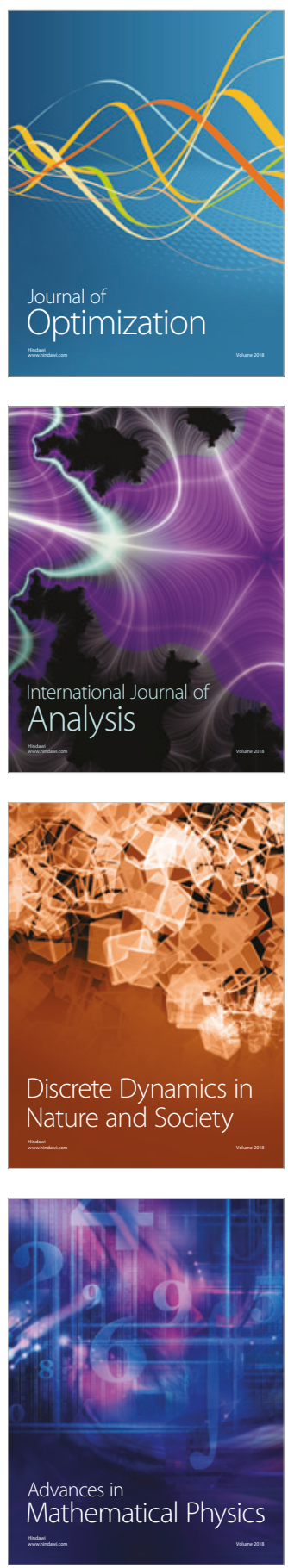